\title{
Combined surface electromyography, near-infrared spectroscopy and acceleration recordings of muscle contraction: The effect of motion
}

\author{
Krista Kauppi \\ Department of Medical Technology \\ University of Oulu, Oulu, 90014, Finland \\ Vesa Korhonen \\ Department of Diagnostic Radiology \\ Medical Research Center, Oulu University Hospital \\ Research Unit of Medical Imaging, Physics and Technology \\ Faculty of Medicine, University of Oulu \\ P. O. Box 50, Oulu, 90029 OYS, Finland \\ Hany Ferdinando \\ Health $\&$ Wellness Measurements Group \\ Optoelectronics and Measurement Techniques Unit \\ University of Oulu, Oulu, 90014, Finland \\ Mika Kallio \\ Department of Clinical Neurophysiology \\ Medical Research Center, Oulu University Hospital \\ Research Unit of Medical Imaging, Physics and Technology \\ Faculty of Medicine, University of Oulu \\ P. O. Box 50, Oulu, 90029 OYS, Finland \\ Teemu Myllylä* \\ Health \& Wellness Measurements Group \\ Optoelectronics and Measurement Techniques Unit \\ University of Oulu, Oulu, 90014, Finland \\ teemu.myllyla@ee.oulu.fi
}

Received 1 April 2016

Accepted 13 October 2016

Published 9 December 2016

${ }^{*}$ Corresponding author.

This is an Open Access article published by World Scientific Publishing Company. It is distributed under the terms of the Creative Commons Attribution 4.0 (CC-BY) License. Further distribution of this work is permitted, provided the original work is properly cited. 


\title{
K. Kauppi et al.
}

\begin{abstract}
Noninvasive techniques, surface electromyography (sEMG) in particular, are being increasingly employed for assessing muscle activity. In these studies, local oxygen consumption and muscle metabolism are of great interest. Measurements can be performed noninvasively using optics-based methods such as near-infrared spectroscopy (NIRS). By combining energy consumption data provided by NIRS with muscle level activation data from sEMG, we may gain an insight into the metabolic and functional characteristics of muscle tissue. However, muscle motion may induce artifacts into EMG and NIRS. Thus, the inclusion of simultaneous motion measurements using accelerometers (ACMs) enhances possibilities to perceive the effects of motion on NIRS and EMG signals.

This paper reviews the current state of noninvasive EMG and NIRS-based methods used to study muscle function. In addition, we built a combined sEMG/NIRS/ACM sensor to perform simultaneous measurements for static and dynamic exercises of a biceps brachii muscle. Further, we discuss the effect of muscle motion in response of NIRS and EMG when measured noninvasively. Based on our preliminary studies, both NIRS and EMG supply specific information on muscle activation, but their signal responses also showed similarities with acceleration signals which, in this case, were supposed to be solely sensitive to motions.
\end{abstract}

Keywords: sEMG; NIRS; accelerometer; motion artifacts; biceps brachii muscle.

\section{Introduction}

Electromyography (EMG) is most commonly used in the field of neurophysiology or electrodiagnostic medicine. ${ }^{1}$ There are multiple ways of gaining data on muscle activity, including intramuscular measurements performed using a needle or a wire. ${ }^{2}$ EMG studies can also be realized using noninvasive techniques, which is also preferable for patients, because intramuscular measurements can be painful and may potentially lead to destruction of muscle fibers. ${ }^{3}$ Surface EMG (sEMG) employs surface electrodes that can be attached to skin overlying the muscle. ${ }^{4}$ The signal is an interference pattern of all the active motor units (MUs) that are the smallest functional groupings in a muscle. ${ }^{2,5} \mathrm{sEMG}$ has been used to study muscle activation, the muscle force and fatigue processes. ${ }^{1,6,7}$

The origin of the sEMG signal is the bio-electric activity of muscle fibers, which is due to ion channel activity (e.g., $\mathrm{Na}^{+}, \mathrm{K}^{+}, \mathrm{Cl}^{-}, \mathrm{Ca}^{2+}$ ) and it can be described as a zero-mean random process, where the standard deviation is comparative to the firing rate and number of active MUs. ${ }^{1,2}$ The recorded signal represents the average potential of the area and appears on the skin with a limited bandwidth, ranging approximately from $10 \mathrm{~Hz}$ to $400 \mathrm{~Hz} \cdot{ }^{8,9}$ The signal amplitude tends to be extremely small as well, from some microvolts to a few millivolts peakto-peak. ${ }^{6,8}$ The electrodes of sEMG record this electrical activity in the muscle or transduce its ionic current. ${ }^{10}$ The electrodes record from a large muscle area, which means that the recorded sEMG signal can be affected by electrode characteristics, including physical dimension, shape, technology and constituent materials as well as the electrode placement and orientation. ${ }^{1,6,9,10}$ Moreover, electrodes have low-signal resolution, a proportionately narrow frequency band and are sensitive to movement artifacts. $^{1,2}$ In addition, the subcutaneous fat layers and high skin layer conductivity cause signal blurring similar to EEG studies. ${ }^{1}$ As the amount of subcutaneous tissue increases, the median frequency decreases. ${ }^{6}$ One important consideration is the electrode-skin impedance, as variations therein may affect the recorded sEMG signal. This can be due to drying of the gel, changes in skin properties or changes in the electrolyte-skin interface. An additional issue is the noise level at the electrode-skin interface, which is highly dependent on skin treatment. ${ }^{10}$ As a result, sEMG often requires some degree of skin preparation such as cleansing the skin of body oils with alcohol, inserting conductive paste or gel, and, in some cases, shaving body hair. ${ }^{2,8,11}$ In some measurements, the outermost layer of the skin is also abraded. ${ }^{11}$

In assessing muscle function, local oxygen consumption and muscle metabolism are often of great interest. Such measurements rely on using opticsbased methods, particularly near-infrared spectroscopy (NIRS). ${ }^{12}$ In addition to studies of brain function, NIRS is being increasingly utilized for the study of muscle function. Three molecules account 
for most of the NIRS light absorption: hemoglobin (both oxygenated hemoglobin ( $\mathrm{HbO}$ ) and deoxygenated hemoglobin $(\mathrm{Hb})$ ), myoglobin $(\mathrm{Mb})$ and cytochrome oxidase c. ${ }^{13} \mathrm{Mb}$, an iron-containing protein found in muscle fibers, is structurally similar to $\mathrm{Hb}$, and their NIRS spectra are very similar. Consequently, it is currently not possible to explicitly distinguish the contributions of $\mathrm{Mb}$ and $\mathrm{Hb}$ to fluctuations of deoxygenation, and deoxygenation in a muscle is commonly represented as their sum: deoxy $[\mathrm{Hb}+\mathrm{Mb}] \cdot{ }^{14}$ Calculation methods for estimating these concentrations in NIRS are based on the modified Beer-Lambert law (MBLL). Being well-known, MBLL has been widely described in numerous publications. ${ }^{14,15}$ The general idea is that the absorption of the tested sample is proportional to the concentration of the absorber, its extinction coefficient and the path length of light traveling through the sample.

Though the effects of muscle oxygenation status on resistance training adaptations, for example, are not fully understood, NIRS has become an increasingly popular choice for monitoring local muscle oxygenation. NIRS reflects the balance of oxygen delivery to working muscles and oxygen consumption in capillary beds, ${ }^{10}$ such measurements are commonly performed, for example, during exercise. ${ }^{19,20}$ At present, muscle activity can also be monitored in real time by portable multi-channel wireless NIRS devices. ${ }^{21}$

Both sEMG and NIRS have a high time resolution, but a rather limited spatial resolution when measuring noninvasively deep within a tissue. For example, NIRS has a depth sensitivity of approximately $1.5 \mathrm{~cm}$ and the spatial resolution of approximately $1 \mathrm{~cm} .{ }^{16}$ A useful method for improving spatial accuracy involves the use of multidetector probes, ${ }^{31}$ which allow measurements of back-scattered light at different distances to be combined in order to obtain scattering and absorbance properties at different tissue depths. ${ }^{32}$ In the same vein, there is a movement towards sEMG recording with two-dimensional (2D) array systems, known as high-density sEMG (HD-sEMG). ${ }^{1}$ In HD-sEMG, muscle activity is monitored with multiple closely placed electrodes that overlie a restricted area of the skin. This allows recording muscle activity in both the temporal and spatial dimension. ${ }^{33}$ HD-sEMG enables pinponting the location of innervation zones with high accuracy, estimating the velocity, length and orientation of muscle fiber conduction, estimating MU locations, decomposing sEMG interference signals and analyzing the properties of single MUs. ${ }^{1}$

Multimodal studies of muscle function are becoming increasingly common, most of them based on a combination of NIRS and EMG. ${ }^{18,22,23}$ Many of these studies focus on investigating the relationship between muscle electrophysiology and oxygenation or hemodynamics ${ }^{24-28}$ or on gathering more reliable information on, for example, neuromuscular and metabolic activity in relation to muscle fatigue or injury. ${ }^{29,30}$ Moreover, wireless hybrid sEMG/NIRS sensors have recently been proposed for multimodal analysis by such researchers as Guo et al. ${ }^{34}$ Surface EMG and accelerometer (ACM) have been previously used for example for sign language recognition, ${ }^{45}$ fatigue assessment, ${ }^{46}$ Parkinson's disease progress assessment ${ }^{47}$ and for monitoring daily activities of patients who have had a stroke. ${ }^{48}$

\section{Effect of Motion Artifacts}

Two kinds of motion artifacts may appear in measurements of muscle function: motions caused by sudden movements, such as swinging of the arm, and tremor generated by the muscle contraction mechanism. ${ }^{49}$ Particularly, in noninvasive and wearable sensors these are present and should be considered in the analysis.

In general, muscle motion may induce motion artifacts into the signal of interest, which can mislead the subsequent analysis. However, removing these artifacts may also lead to loss of useful data. Most artifact removal methods focus on motions caused by sudden movements, which are commonly present in noninvasive and wearable applications. For example, proposing ACM-based motion artifact removal (ABAMAR), Virtanen et al. ${ }^{52}$ applied a $2 \mathrm{D}$ ACM to detect the presence of motion in sleeping subjects. They sought to correct the baseline shift of the NIRS signal and used a threshold, which was in the rate of acceleration change, to detect movement. On detecting a movement, a flag is raised for a time interval Tm. However, if Tm is less than $1 \mathrm{~s}$, the system sees no movement. To correct the baseline shift, the mean of the NIRS signal before and after Tm are calculated separately to obtain $\bar{A}_{\text {before }}$ and $\bar{A}_{\text {after }}$. If the absolute difference between $\bar{A}_{\text {before }}$ and $\bar{A}_{\text {after }}$ exceeds 2.6 times the SD before TM, a baseline shift change is identified. The amplitude of the signal within Tm is set to $\bar{A}_{\text {before }}$, 
while that of the signal after Tm is multiplied by $\bar{A}_{\text {before }} / \bar{A}_{\text {after. }}$. In another strand of research, Molavi et $a l^{41}$ applied wavelet transform to remove motion artifacts from muscle NIRS measurements without using an ACM. This type of wavelet analysis produces coefficients that make motion artifacts appear as isolated large coefficients in the wavelet domain. To counter that, coefficients which do not belong to the original signal distribution are removed by a statistical test at a specific probability level. This probability is the tuning parameter of the proposed algorithm.

Effect of motion artifacts caused by muscle contraction itself have been studied to a lesser extent. Using state-of-the-art, technology can minimize the influence of some extrinsic noise sources as power line noise and cable motion artifact as well as intrinsic thermal noise originating from the electronics of the amplification system. In order to reduce the noise components of the signal, Luca et al. ${ }^{50}$ proposed to include a high-pass filter to sEMG systems, which reduces baseline noise and the motion artifact from the movement without removing too much of the low-frequency components. Nevertheless, the choice of right high-pass corner frequency depends on application and muscle. Raez et $a l .{ }^{51}$ also suggest that a way to reduce motion artifact is by designing the electronic circuitry and set-up properly. Signal conditioning can also be used in motion artifact reduction. Common ways are the use of adaptive and wavelet filters. ${ }^{2}$ In our approach, we pursued to fatigue the muscles and cause muscle tremor. Surprisingly, we did not find articles about the role of muscle tremor to the motion artifacts.

In the following, we present a multimodal sensor built for performing simultaneous measurements with sEMG, NIRS and ACM. The aim was to study the effect of motion in isometric and isotonic exercises targeting the biceps brachii muscle. A muscle contraction is described as isometric when the muscle contracts without a change in its length, and isotonic when muscle length changes, while the contraction force remains the same. ${ }^{52}$

\section{Measurement Setup and Material}

\subsection{The measurement device}

For measurements of sEMG and acceleration, we utilized a noncommercial prototype produced by
Fibrux. ${ }^{53}$ The prototype consists of a sEMG sensor and inbuilt three-dimensional (3D) acceleration sensor. The cut-off frequencies for the sEMG sensor are $15 \mathrm{~Hz}$ and $250 \mathrm{~Hz}$ and for the ACM $10 \mathrm{~Hz}$. The prototype by Fibrux was integrated with a NIRS device, built in the Optoelectronics and Measurement Techniques Unit, University of Oulu. The NIRS device utilizes a commonly known method, lock-in amplification, which is closely related to the frequency-domain NIRS technique. ${ }^{35} \mathrm{In}$ lock-in amplification, instead of amplification of the received signal over the whole frequency band of interest, only amplitude-modulated light signals at a specific narrow-band reference frequency are amplified. As a result, a significant increase can be achieved in signal-to-noise performance. When an amplified reference signal is multiplied by the lockin reference signal and then low-pass filtered, intensity changes in the remaining low-pass filtered output signal correlate with attenuation changes in the received light signal. Furthermore, the lock-in amplification technique enables separation of several signals from each other. Thus, it is possible to label each wavelength of light for a specific modulation frequency, illuminate all wavelengths simultaneously and then separate them in the receiver. ${ }^{35}$ Normally, this technique requires multi-distance recordings to fit a linear regression of distance versus changes of phase, to accurately estimate hemodynamics derived from the fitted slopes. ${ }^{36}$ However, mainly because of the large electrode size of sEMG sensors, we decided to use a single distance pair of optodes, located at $3 \mathrm{~cm}$ from each other, with two EMG electrodes placed between the optodes, as shown in Fig. 1. Although this type of single distance technique has its limitations, it is used in most NIRS-based muscle studies. ${ }^{17}$ Temporal oxygenation changes were calculated at the wavelengths of $660 \mathrm{~nm}$ and $830 \mathrm{~nm}$. In our NIRS, sEMG and acceleration recordings, the sampling rate was $500 \mathrm{~Hz}$, and synchronization between the different modalities was provided by a trigger pulse.

$\mathrm{HbO}, \mathrm{Hb}+\mathrm{Mb}$ and total deoxy $\mathrm{t}(\mathrm{Hb}+\mathrm{Mb})$ were calculated from raw NIRS time courses using MATLAB's HomER2 NIRS-processing package. ${ }^{35}$ A differential pathlength factor (DPF), needed for the calculation, is determined by the absolute optical pathlength divided by the known distance between optodes. The value of DPF is dependent on the place of measurement. In the case of the adult forearm, for example, its mean value is $3.59 \pm 0.32$ 


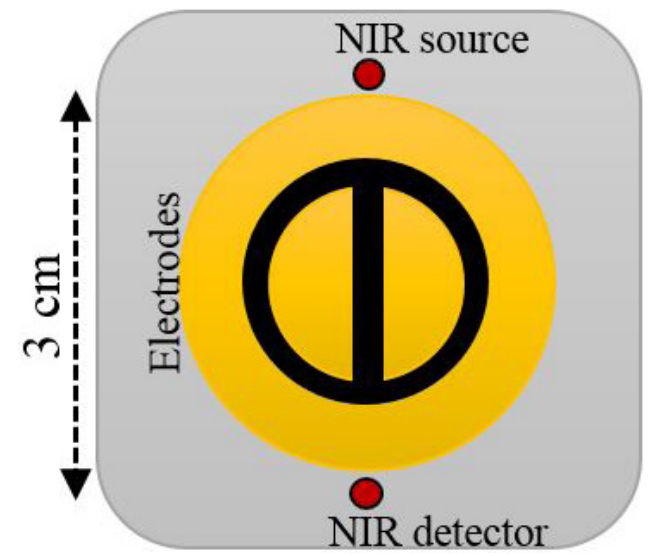

Fig. 1. Illustration of the sensor. It includes an optode consisting of a fiber optic near-infrared (NIR) light source at a distance of $3 \mathrm{~cm}$ from the fiber optic detector, both with a fiber thickness of $2.5 \mathrm{~mm}$; a built-in 3D ACM and two copper electrodes with a ground plate placed between the optode. Thus, NIRS and sEMG measured the average activity of the muscle from approximately the same area. This noncommercial sEMG sensor prototype was produced by Fibrux.

for both male and female subjects, while the value for the adult brain is $5.93 \pm 0.43 .{ }^{37}$ On the other hand, Duncan et al. have reported separate DPF values for males and females as a function of wavelength. At $832 \mathrm{~nm}, \mathrm{DPF}$ was $3.53 \pm 0.55$ for males and $4.34 \pm 0.78$ for females. At $690 \mathrm{~nm}$, the $\mathrm{DPF}$ value for males was $3.96 \pm 0.62$, while that of females was $4.91 \pm 0.81$. Why females have a statistically significant higher forearm DPF than males is probably due to a thicker fat layer. ${ }^{38}$ Nonetheless, DPF is still a constant value which influences only the absolute values of $\mathrm{Hb}, \mathrm{HbO}$ and $\mathrm{HbT}{ }^{39}$

\subsection{Subjects and performed task}

The sensor was placed on the subjects' right biceps brachii (Fig. 2.) and fixed in place with an elastic band. Five healthy volunteers participated this study, performing three exercises (static, dynamic and isometric) targeting the biceps brachii muscle. Subjects: 3 males (age 27 years, height $192 \mathrm{~cm}$, weight $73 \mathrm{~kg}$ ); (age 33 years, height $191 \mathrm{~cm}$, weight $103 \mathrm{~kg}$ ); (age 44 years; height $189 \mathrm{~cm}$; weight $85 \mathrm{~kg}$ ) and 2 females: (age 23 years; height $170 \mathrm{~cm}$; weight $62 \mathrm{~kg}$ ) and (age 27 years; height $172 \mathrm{~cm}$; weight $72 \mathrm{~kg}$ ). Static and dynamic exercises were conducted on the same day, while the isometric exercise took place a week later. A static exercise was always conducted first, followed immediately by a dynamic exercise. The purpose was to fatigue the muscle in order to examine correlations between sEMG and NIRS data, and to observe the effect of muscle motion recorded simultaneously by an ACM. In both static and dynamic exercises, the subject was sitting in a chair with their arm on a table. No weights were used, since we wanted to study the effect of natural muscle power. The subjects were instructed to tense the muscle for $10 \mathrm{~s}$ and then relax it for $20 \mathrm{~s}$. This protocol was repeated five times. In the dynamic task, the subjects did five sets of five braking contractions of biceps brachii at maximal power, with a $20 \mathrm{~s}$ rest between the sets. In the isometric exercise, the subjects first stood straight and then leaned against a wall while holding a customized $5 \mathrm{~kg}$ weight at an elbow angle of $90^{\circ}$ for as long as possible. This isometric exercise was repeated after 1 min break.

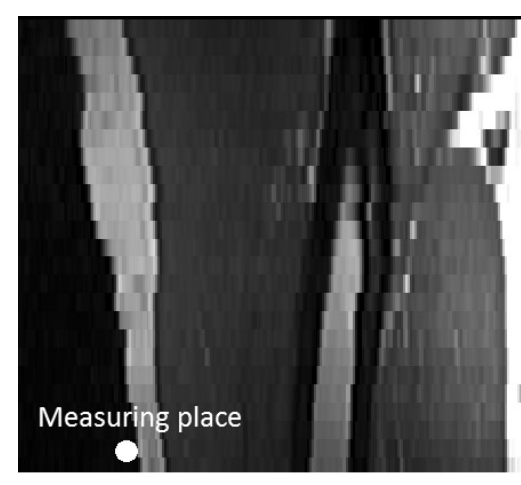

(a)

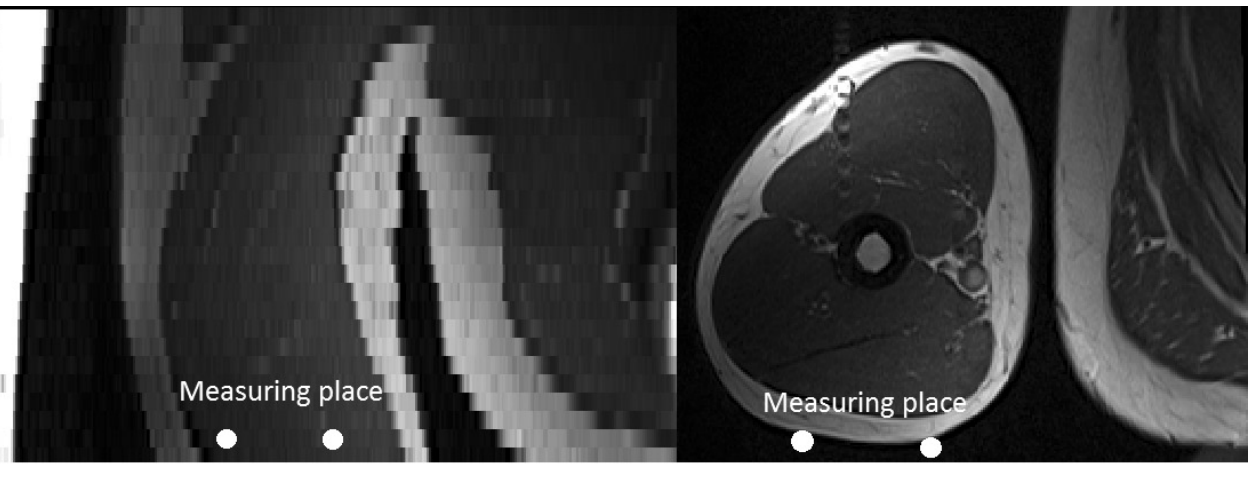

(b) (c)

Fig. 2. Anatomical image of the arm of Subject 4. The measuring place was on the right biceps brachii, illustrated with white circles in the anatomical image in (a) sagittal (b) coronal and (c) axial slices. An important challenge for sEMG and NIRS is the fact that soft tissue (mostly fat), shown in white color, intervenes with the signal, especially when the active muscle is $10 \mathrm{~mm}$ or more below skin surface. ${ }^{1}$ 


\section{K. Kauppi et al.}

In our experiments, our aim was to study the motions caused by the muscle contraction itself. Thus, the subjects were advised to avoid other movements than flexing the biceps brachii muscle.

\section{Results}

SEMG signals can be evaluated both in the time and frequency domains. Among the most common time-domain methods are root mean square (RMS) and average muscle fiber conduction velocity $(\mathrm{CV})$. In the frequency domain, the focus of attention is on the median frequency of the power density function. ${ }^{40}$ Frequency domain measurements can be useful in fatigue assessment, as fatigue causes a shift in median frequency.

In the following Figs. 3-6, we chose to survey the signals in time domain in order to demonstrate the clear correspondence between the three modalities in contrast to muscle contraction. All presented signals are measured from right biceps brachii (see Fig. 2) of Subject 4. The responses of the other subjects were similar. Shown are deoxy $(\mathrm{Hb}+\mathrm{Mb})$, original data (blue) and upper envelograms (red) of synchronized sEMG and ACM signals, taken with 100 point smoothing. Additionally, the signals are normalized to scale 0 to 1 using Origin 2015 version from Originlab. Deoxy $(\mathrm{Hb}+\mathrm{Mb})$ were obtained from the NIRS data using MATLAB. The spectral range of all presented envelope signals is approximately between $0-1.8 \mathrm{~Hz}$, thus shown are lower signal fluctuations caused by muscle contraction and motions. In addition, higher frequency transients for EMG and ACM are included with the envelopes, except in Figs. 5 and 6.

In the performed experiments, test subjects found it difficult to control the contraction of the muscle accurately or to repeat the contraction equally, thus such duration and the strength of contraction constantly varied. Thus, statistical analysis in this case would not be relevant. Thereby, in the following, we show only examples of signal responses from single subjects.

Figure 3 shows an effect of four static muscle contractions. The muscle contraction causes a simultaneous response in $\mathrm{ACM}$, muscle deoxy $(\mathrm{Hb}+$ $\mathrm{Mb})$ concentration with the activation of MUs, visible in sEMG.

Figure 4 shows signal responses for dynamic contractions. In dynamic exercise, we can see steep spikes both in deoxy $(\mathrm{Hb}+\mathrm{Mb})$ and sEMG signals. The deoxy $(\mathrm{Hb}+\mathrm{Mb})$ concentration increases
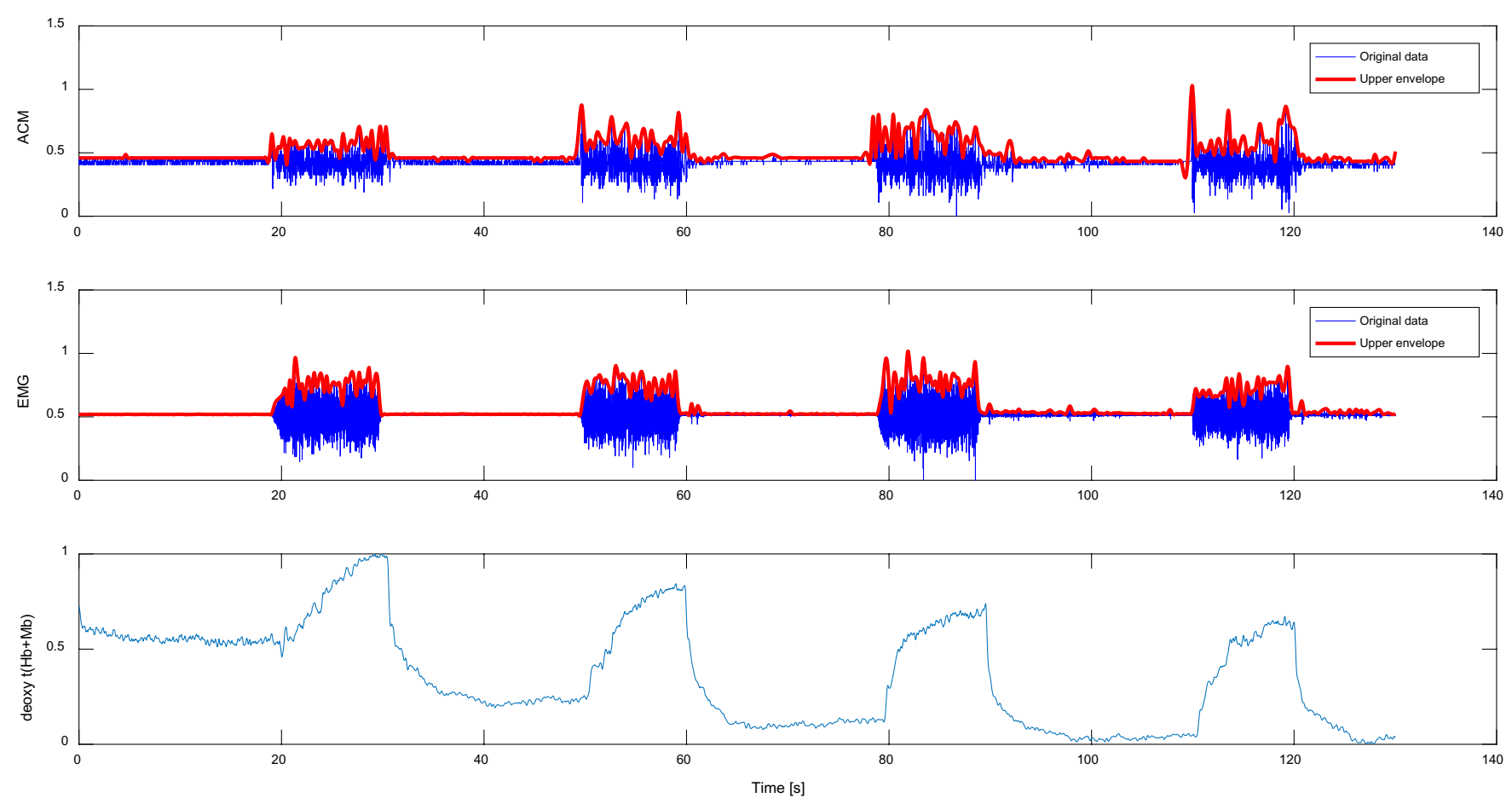

Fig. 3. Signal responses from ACM, deoxy $(\mathrm{Hb}+\mathrm{Mb})$ and sEMG during a static exercise. There is a strong fluctuation in all the three signals caused by the four static contractions. 

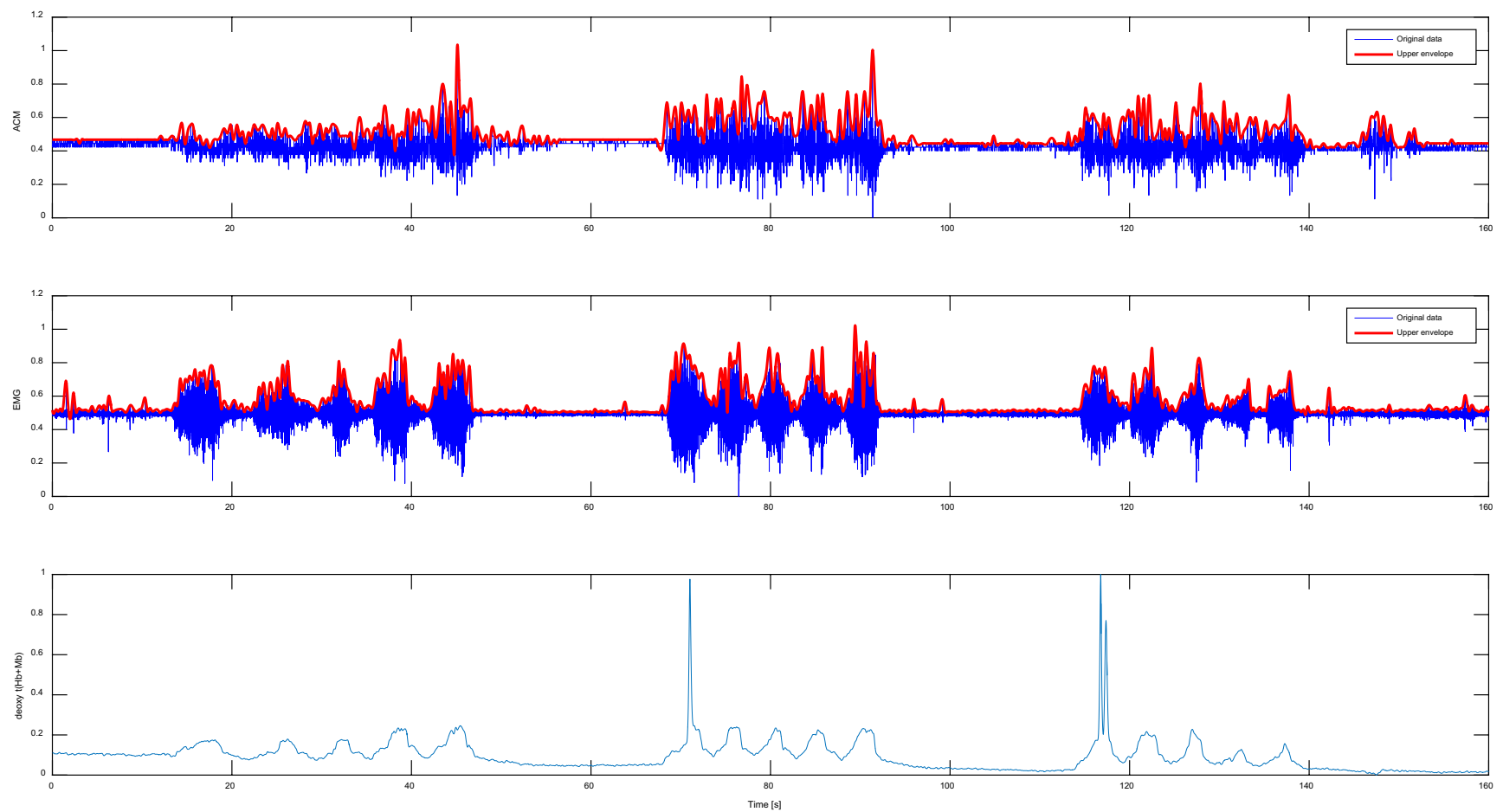

Fig. 4. Signals of ACM, deoxy $(\mathrm{Hb}+\mathrm{Mb})$ and sEMG during dynamic exercise. We can see that the ACM signal also fluctuates between the dynamic contractions, and also after the third sets of contractions. This, however, is not visible in deoxy (Hb $+\mathrm{Mb})$ and sEMG signals which indicates that deoxy $(\mathrm{Hb}+\mathrm{Mb})$ and sEMG are more sensitive to muscle contraction.

steeply and receives the maximum value at the same time as sEMG signal has its maximum. There are high spikes at $70 \mathrm{~s}$ and $117 \mathrm{~s}$ which are easily to spot at the beginning of the short braking contraction series. However, this motion artifact was not seen in EMG and ACM measurements.

Figures 5 and 6 show responses for isometric contractions when continuously holding up a $5 \mathrm{~kg}$
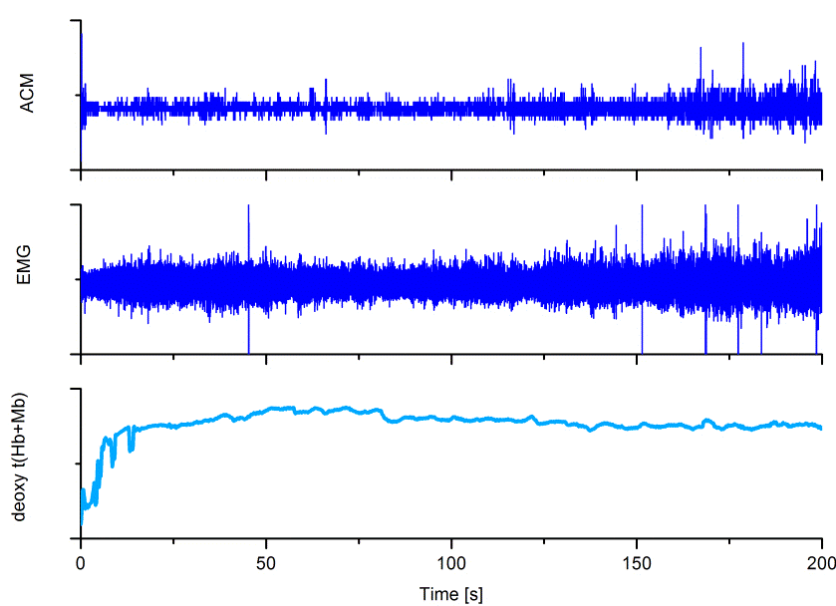

Fig. 5. The response of the first isometric contraction of Subject 4 . Subject 4 holds up the $5 \mathrm{~kg}$ weight for $200 \mathrm{~s}$. weight as long as possible, followed by a $1 \mathrm{~min}$ break before the second contraction. In the beginning, the $\mathrm{ACM}$ and EMG signals are quite stable but as the fatigue starts to increase, due to the long
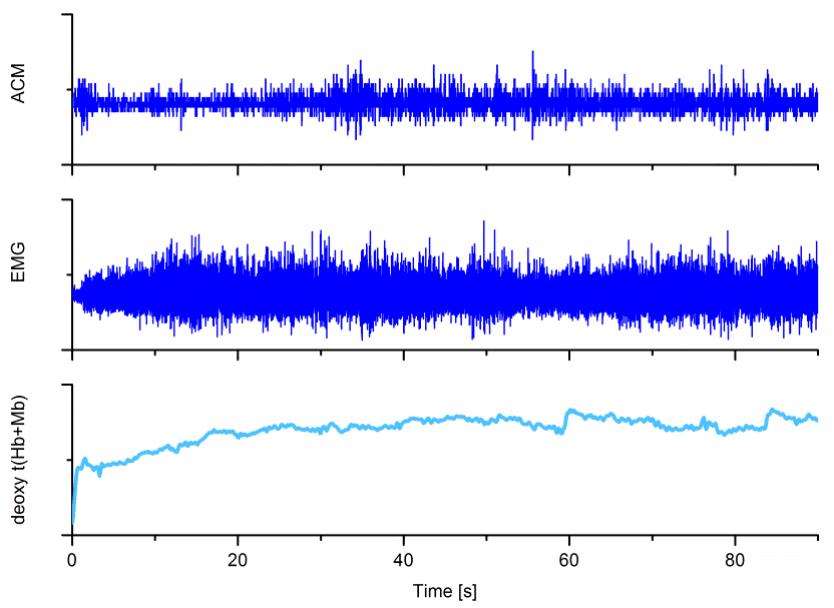

Fig. 6. The response of the second isometric contraction of Subject 4 . In the second contraction, Subject 4 could hold up the $5 \mathrm{~kg}$ weight only for $90 \mathrm{~s}$ whereas in the first contraction duration was $200 \mathrm{~s}$. We can see that due to the fatigue caused by the first isometric exercise, the tremor effect in ACM as well as in EMG is more present in the second than in the first isometric contraction. Contraction causes increase in deoxy $(\mathrm{Hb}+$ $\mathrm{Mb})$ and fluctuates a little more when fatique starts. 


\section{K. Kauppi et al.}

isometric contraction, more muscle tremor is present, particularly in Fig. 5 between $160 \mathrm{~s}-200 \mathrm{~s}$, and in the second contraction, Fig. 6, already between $30 \mathrm{~s}-90 \mathrm{~s}$. Moreover, deoxy $(\mathrm{Hb}+\mathrm{Mb})$ slightly fluctuates. The signal behavior varies along the test subjects, however, with the same main character as presented here.

\subsection{The effect of motion}

\section{(a) Spikes in signal}

Many higher spikes in ACM, visible in static and dynamic exercises, were most likely caused by sudden muscle motions. These, however, were lesser visible in corresponding sEMG and NIRS signals which positively indicates that sEMG and NIRS are more sensitive to muscle contraction rather than its sudden motions. Interestingly, on the other hand, the dynamic experiment measurement, in Fig. 4, shows motion artifact induced to NIRS as high spikes at $70 \mathrm{~s}$ and $117 \mathrm{~s}$ which are easily to spot at the beginning of the short braking contraction series. Mostly likely, the spikes in NIRS are caused by the movements of the optode, because these are only visible in NIRS signal. Thus, we cannot utilize data from ACM to remove the artifact, for example, with the method proposed by Virtanen et al. ${ }^{52}$ However, there is still possibility to remove this artifact using algorithm proposed by Molavi et al. ${ }^{40}$ because it requires signal from NIRS measurement only. Utilizing this algorithm, the first spike on NIRS signal could be eliminated, as seen in Fig. 7, but the shape of the NIRS signal changed a bit. However, the second spike could be removed completely but the algorithm can attenuate it.

\section{(b) Muscle tremor}

During contraction, the muscle produces constant tremor generated by the muscle contraction mechanism. ${ }^{48}$ This is clearly visible in ACM signal in all Figs. 3-6, as well as in Figs. 8 and 9. However, how this affects the EMG and NIRS signals should be more studied. In Fig. 8 is shown the tremor effect in ACM signal and corresponding EMG signal, at spectrum range of $15 \mathrm{~Hz}-250 \mathrm{~Hz}$. When compared the spectrum between rest and contraction, there is obviously a notable difference in the power density. Moreover, during dynamic contraction, shown in
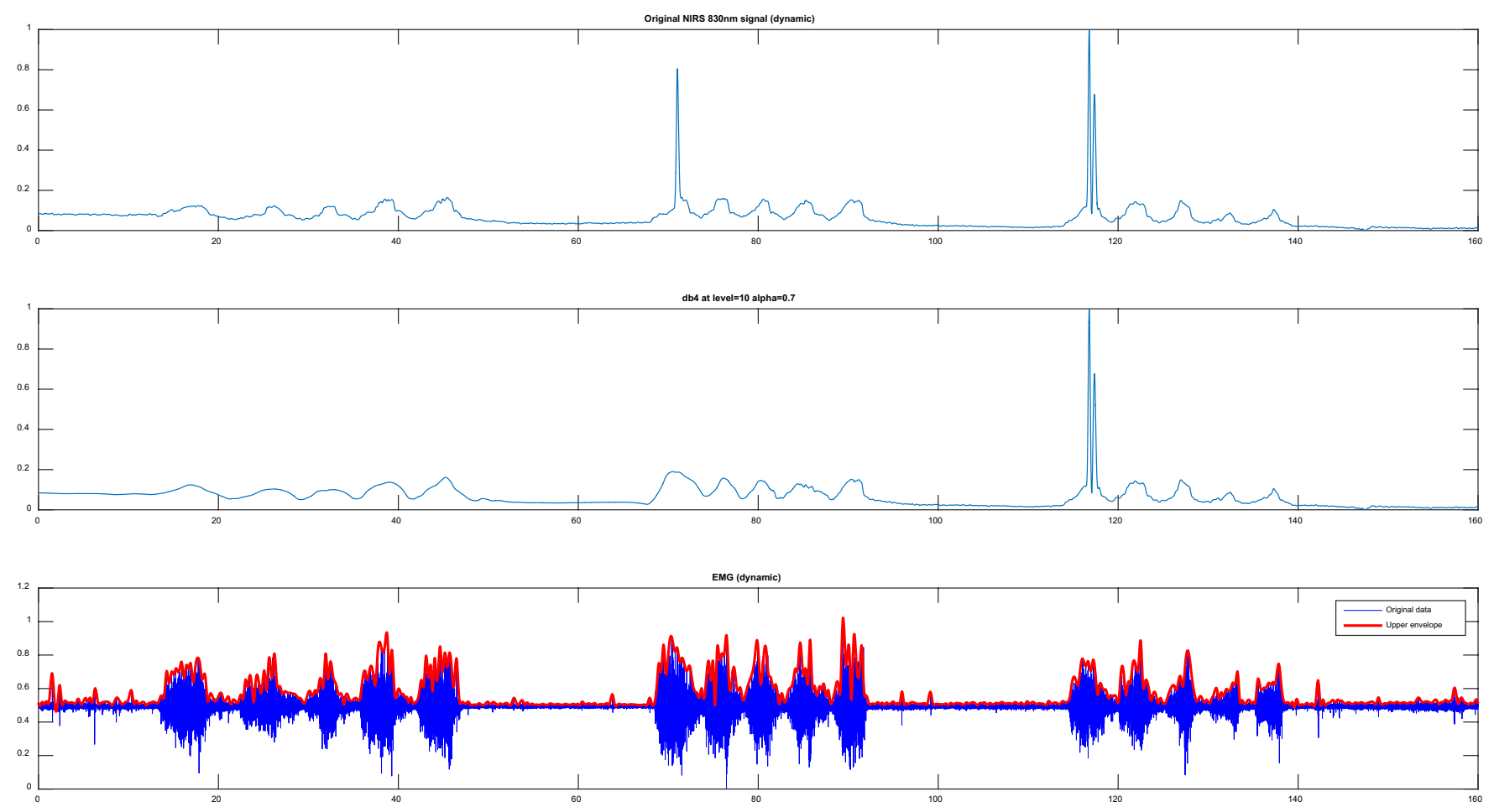

Fig. 7. Applying motion artifact removal to dynamic experiment. Top: original NIRS signal, middle: NIRS signal after removing motion artifact, bottom: EMG signal. 


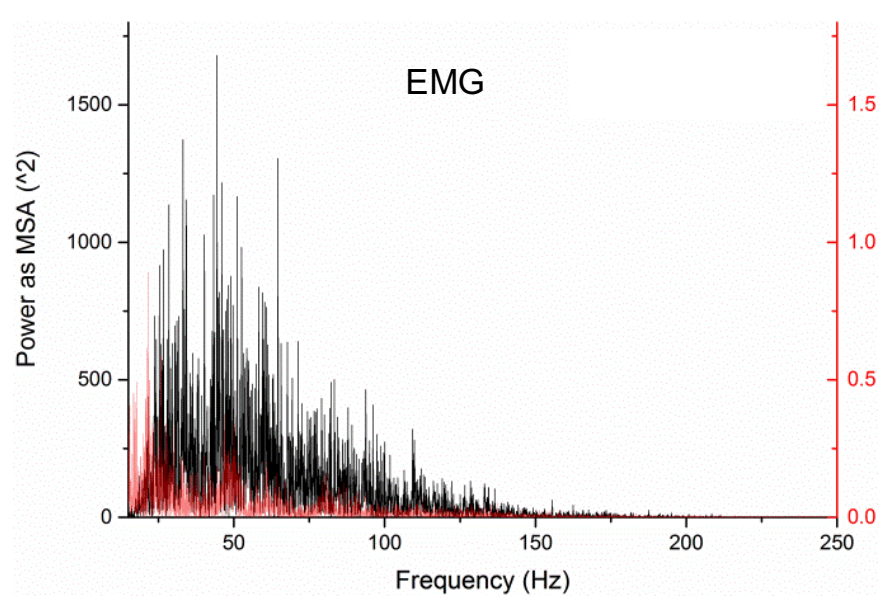

(a)

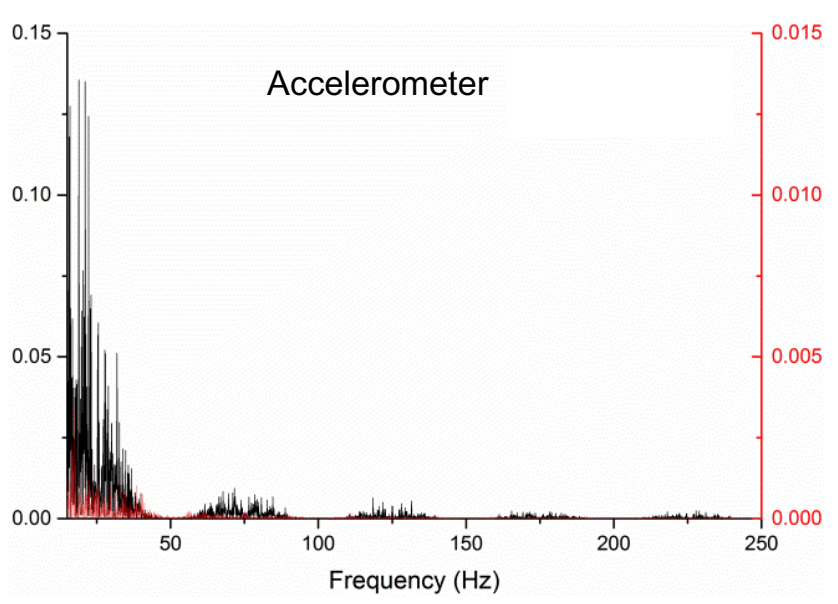

(b)

Fig. 8. Power spectra of EMG (a) and ACM (b) during rest (red) versus static contraction (black). During contraction, the power spectra of ACM is visible up to $40 \mathrm{~Hz}$ and in EMG up to $150 \mathrm{~Hz}$.

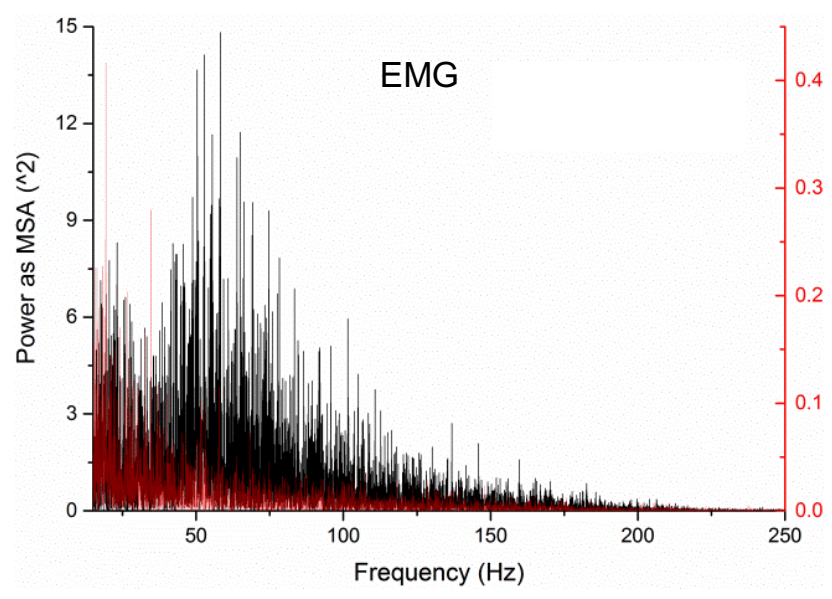

(a)

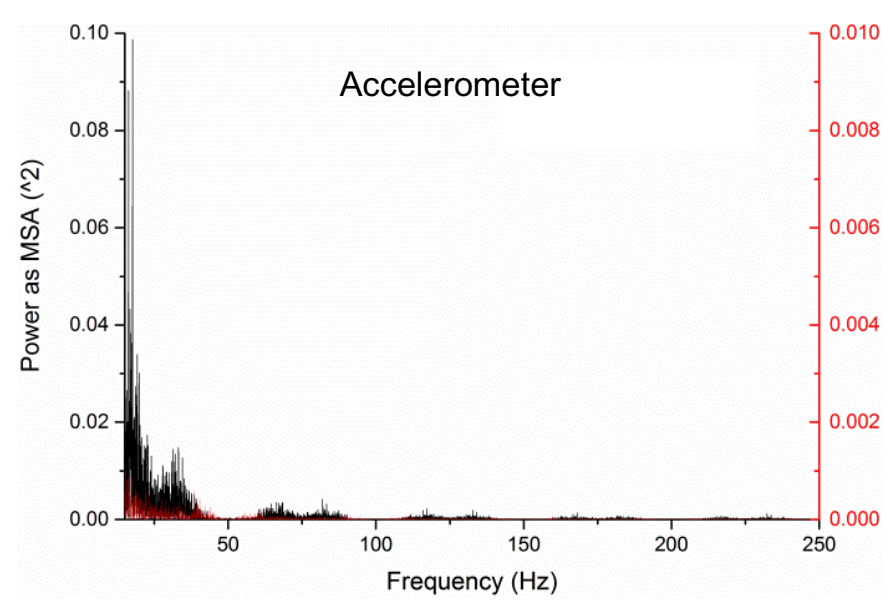

(b)

Fig. 9. Power spectra of EMG (a) and ACM (b) during rest (red) versus dynamic contraction (black). During dynamics contraction, the power spectra of ACM similarly appears in the frequency range up to $40 \mathrm{~Hz}$. However, in EMG it widens up to $200 \mathrm{~Hz}$.

Table 1. Correlation between ACC and EMG during static and dynamics exercises.

\begin{tabular}{lrr}
\hline $\begin{array}{l}\text { Correlation between } \\
\text { ACC and EMG }\end{array}$ & Static & Dynamic \\
\hline Subject 1 & 0.04119 & 0.29304 \\
Subject 2 & 0.17461 & 0.53191 \\
Subject 3 & 0.22306 & -0.20109 \\
Subject 4 & 0.14402 & 0.09474 \\
Subject 5 & 0.29885 & 0.39688 \\
Average & 0.17635 & 0.22310 \\
STD & 0.09553 & 0.28586 \\
\hline
\end{tabular}

Fig. 9, the difference is greater, particularly in EMG. In addition, we noticed clear shift in median frequency of the power density, when compared the first contraction to the last contraction of the exercise. This was in accordance to the commonly known phenomenon that fatigue causes a shift in median frequency. ${ }^{40}$

Table 1 shows correlations between acceleration signal and corresponding EMG signal during static and dynamic exercises. The average correlations for five subjects is 0.18 (static) and 0.22 (dynamic). 


\section{Discussion}

Presented results obviously require statistical validation since only five subjects participated in the experiments. Though, presented setup, combining sEMG, NIRS and ACM, show the potential for muscle studies since it can easily distinguish in all signals, even when the muscle is either in relax or in muscle contraction. In addition, muscle tremor as well as sudden spikes caused by motion were clearly visible in acceleration signal. But, there is still a need to find an exact relationship between a given physiological event and a specific sEMG parameter that could be supported by NIRS and ACM data. Our results show that spikes caused by sudden muscle motions were visible in ACM, however, to a lesser degree in EMG and NIRS. As expected, dynamics exercise causes more motion in the muscle, in contrast to static. In addition, in dynamics exercise, there was also motion present between the contractions, during rest, however, this introduced no noise to EMG and NIRS signals. In general, muscle tremor below $40 \mathrm{~Hz}$ was significant during contraction in all experiments, as can be seen in Figs. 8 and 9. Interestingly, static and dynamic contractions seem to cause similar frequency response in power spectra of acceleration. In EMG, dynamic contraction causes wider power spectra. The average correlations between ACM and EMG for five subjects was 0.18 in static contraction and 0.22 in dynamic contraction. Hence, dynamic contractions seem to affect EMG signal more than static. In accordance with the previous studies, presented results show that muscle motions induce artifacts into both EMG and NIRS signals. Artifact removal methods, for example, the methods presented by Molavi ${ }^{41}$ and Virtanen ${ }^{52}$ can be potentially used, in particular, when the signal analysis is based on multimodal data, gathered using such presented measurement device.

According to Elcadi ${ }^{12}$ in healthy subjects, there is an inverse linear relationship between NIRS and EMG signals, meaning that as EMG amplitude increases, oxygenation decreases. ${ }^{1}$ This correlation between increasing load, EMG and $\mathrm{VO}_{2}$ has also been found in other studies, ${ }^{40-44}$ which indicates that muscle force increases as a function of load. ${ }^{42}$ Changes in EMG amplitude are mainly caused by a change in the number of active MUs and their mean firing rate. ${ }^{40}$ Our results show that as the EMG amplitude increases, there is a simultaneous increase in deoxy concentration $(\mathrm{Hb}+\mathrm{Mb})$, see Figs. 3-6. Moreover, Figs. 5 and 6 indicate that the tremor in $\mathrm{ACM}$ as well as in EMG, caused by the isometric contraction, increases due to fatique, while deoxy $(\mathrm{Hb}+\mathrm{Mb})$ starts to slightly fluctuate.

Despite clear advantages offered by the sEMG technique, it is difficult to integrate into routine clinical use due to a lack of standards for sensors, configurations, electrode placements and recording protocols. Nonetheless, the utility of sEMG as a tool of examining neuromuscular function is obvious. Moreover, there is an ongoing effort to standardize and validate sEMG. A case in point in Europe is SENIAM (surface EMG for the noninvasive assessment of muscles). Also the International Society of Electromyography and Kinesiology (ISEK) has put forward recommendations regarding the use of sEMG techniques and methodologies. ${ }^{3}$

\section{Conclusions}

A combination of these three noninvasive methods offers valuable insights for the study of muscle function. In addition to energy consumption data provided by NIRS, we also gain knowledge about muscle level activation using sEMG, and the effect of motion on these two signals. However, the exact relationship between the modalities and physiological event still need further studies.

SEMG is an intriguing research area and, combined with NIRS and ACM, it can deliver multiple benefits. For example, HD-sEMG yields new information about the central nervous system and MUs. Moreover, as current clinical methods can be painful, they render large studies practically impossible. A noninvasive technique, in contrast, would increase the number of available test subjects, allowing researchers and clinicians to reap major benefits.

\section{References}

1. D. Stegeman, J. Blok, H. Hermens, K. Roeleveld, "Surface EMG models: Properties and applications," J. Electromyogr. Kinesiol. 10, 313-326 (2000).

2. E. Clancy, E. Morin, R. Merletti "Sampling, noisereduction and amplitude estimation issues in surface electromyography," J. Electromyogr. Kinesiol. 12, $1-16$.

3. J. Hogrel, "Clinical application of surface electromyography in neuromuscular disorders," Neurophysiol. Clin. 35, 59-71 (2005). 
4. S. Pullman, D. Goodin, A. Marquinez, S. Tabbal, M. Rubin, "Clinical utility of surface EMG," Neurology 55, 171-177 (2000).

5. R. Merletti, A. Holobar, D. Farina, "Analysis of motor units with high-density surface electromyography," J. Electromyogr. Kinesiol. 18, 879-890 (2008).

6. C. De Luca, "The use of surfacce electromyography in biomechanics," J. Appl. Biomech. 13, 135-163 (1997).

7. O. Sayli, A. Akin, H. Cotuk, "Correlation analysis between surface electromyography and continuouswave near-infrared spectroscopy parameters during isometric exercise to volitional fatigue," Turkish J. Electr. Eng. Comput. Sci. 22, 780-793 (2014).

8. M. Cavalcanti, T. Vieira, "Surface electromyography: Why, when and how to use it," Rev. Andaluza Med. Dep. 4(1), 17-28 (2011).

9. R. Merletti, A. Rainoldi, D. Farina, "Surface electromyography for noninvasice characterization of muscle," Exerc. Sport Sci. Rev. 29, 20-25 (2011).

10. R Merletti, A. Botter, A Troiano, E Merlo, M Minetto, "Technology and instrumentation for detection and conditioning of the surface electromyographic signal: State of the art," Clin. Biomech. 24, 122-134 (2009).

11. S. Roy, G. De Luca, M. Cheng, A. Johansson, L. Gilmore, C. De Luca, "Electro-mechanical stability of surface EMG sensors," Med. Bio. Eng. Comput. 45, 447-457 (2007).

12. G. Elcadi, Near infrared spectroscopy for assessing oxygenation and hemodynamics in the upper extremities of healthy subject and patients with workrelated muscle pain, Thesis, Department of Community Medicine and Rehabilitation, Rehabilitation medicine, Umeå University (2012).

13. R. Boushel, C. A. Piantadosi, "Near-infrared spectroscopy for monitoring muscle oxygenation," Scand. J. Med. Sci. Sports 11(4), 213-222 (2001).

14. B. M. Celie, J. Boone, J. Dumortier, W. Derave, T. De Backer and J. G. Bourgois, "Possible influences on the interpretation of functional domain (FD) near-infrared spectroscopy (NIRS): An explorative study," Appl. Spectrosc. 70(2), 363-371 (2016).

15. M. Cope, "The application of near infrared spectroscopy to noninvasive monitoring of cerebral oxygenation in the newborn infant," Dep. Med. Phys. Bioeng. 342 (1991).

16. M. Cope, D. Delpy, "System for long-term measurement of cerebral blood and tissue oxygenation on newborn infants by near infra-red transillumination," Med. Biol. Eng. Comput. 26(3) 289-294 (1988).

17. M. Ferrari, M. Muthalib, V. Quaresima, "The use of near-infrared spectroscopy in understanding skeletal muscle physiology: Recent developments," Philos.
Trans. R. Soc. London A: Math., Phys. Eng. Sci. 369(1955), 4577-4590 (2011).

18. B. R. Scott, K. M. Slattery, D. V. Sculley, R. G. Lockie, B. J. Dascombe, "Reliability of telemetric electromyography and near-infrared spectroscopy during high-intensity resistance exercise," J. Electromyogr. Kinesiol. 24(5), 722-730 (2014).

19. F. Felici, V. Quaresima, L. Fattorini, P. Sbriccoli, G. C. Filligoi, M. Ferrari, "Biceps brachii myoelectric and oxygenation changes during static and sinusoidal isometric exercises," J. Electromyogr. Kinesiol. 19(2), e1-e11 (2009).

20. Y. N. Bhambhani, "Muscle oxygenation trends during dynamic exercise measured by near infrared spectroscopy," Can. J. Appl. Physiol. 29(4), 504-523 (2004).

21. P. Yao, W. Guo, X. Sheng, D. Zhang, X. Zhu, A portable multi-channel wireless NIRS device for muscle activity real-time monitoring. In engineering in medicine and biology society (EMBC), 2014 IEEE 36th Ann. Int. Conf., pp. 3719-3722.

22. Y. Bhambhani, J. L. Fan, N. Place, J. RodriguezFalces, B. Kayser, "Electromyographic, cerebral, and muscle hemodynamic responses during intermittent, isometric contractions of the biceps brachii at three submaximal intensities," Front. Physiol. 5(190), (2014).

23. T. E. Cayot, J. D. Lauver, C. R. Silette, B. W. Scheuermann, "Effects of blood flow restriction duration on muscle activation and microvascular oxygenation during low-volume isometric exercise," Clin. Physiol. Funct. Imag. Jan 7 (2015), doi: 10.1111/cpf.12228.

24. C. Booghs, S. Baudry, R. Enoka, J. Duchateau, "Influence of neural adjustments and muscle oxygenation on task failure during sustained isometric contractions with elbow flexor muscles," Exp. Physiol. 97(8), 918-929 (2012).

25. G. H. Elcadi, M. Forsman, U. Aasa, M. Fahlstrom, A. G. Crenshaw, "Shoulder and forearm oxygenation and myoelectric activity in patients with work-related muscle pain and healthy subjects," Eur. J. Appl. Physiol. 113(5), 1103-1115 (2013).

26. S. A. Ferguson, W. G. Allread, P. Le, J. Rose, W. S. Marras, "Shoulder muscle fatigue during repetitive tasks as measured by electromyography and nearinfrared spectroscopy," Hum. Factors 55(6), 10771087 (2013).

27. W. Moalla, A. Merzouk, F. Costes, Z. Tabka, S. Ahmaidi, "Muscle oxygenation and EMG activity during isometric exercise in children," J. Sport. Sci. 24(11), 1195-1201 (2006).

28. E. Horikoshi, H. Ishikawa, T. Yoshida, S. Tamaoki, T. S. Kajii, "Oxygen saturation and electromyographic changes in masseter muscle during experimental 
chewing of gum with harder texture," Acta Odontol. Scand. 71(6), 1378-1385 (2013).

29. W. J. Albert, G. G. Sleivert, J. P. Neary, Y. N. Bhambhani, "Monitoring individual erector spinae fatigue responses using electromyography and near infrared spectroscopy," Can. J. Appl. Physiol. 29(4), 363-378 (2004).

30. R. T. Kell, Y. Bhambhani, "Relationship between erector spinae muscle oxygenation via in vivo near infrared spectroscopy and static endurance time in healthy males," Eur. J. Appl. Physiol. 102(2), 243-250 (2008).

31. D. A. Boas, A. M. Dale, Franceschini, "Diffuse optical imaging of brain activation: Approaches to optimizing image sensitivity, resolution, and accuracy," Neuroimage, 23, S275-S288 (2004).

32. V. O. Korhonen, T. S. Myllyla, M. Y. Kirillin, A. P. Popov, A. V. Bykov, A. V. Gorshkov, V. Kiviniemi, "Light propagation in NIR spectroscopy of the human brain," IEEE J. Sel. Top. Quantum Electron. 20(2), 289-298 (2014).

33. G. Drost, D. Stageman, B. Engelen, M. Zwarts, "Clinical applications of high-density surface EMG: A systematic review," J. Lectromyogr. Kinesiol. 16, 586-560 (2006).

34. W. Guo, X. Sheng, H. Liu, X. Zhu, Development of a multi-channel compact-size wireless hybrid sEMG/NIRS sensor system for prosthetic manipulation. IEEE Sensor. 16(2), 447-456 (2016).

35. H. S. S. Sorvoja, T. S. Myllylä, M. Y. Kirillin, E. A. Sergeeva, R. A. Myllylä, A. A. Elseoud, V. Kiviniemi ,"Non-invasive, MRI-compatible fibreoptic device for functional near-IR reflectometry of human brain," Quantum Electron. 40(12), 1067 (2010).

36. S. Fantini, M. A. Franceschini, E. Gratton, "Semiinfinite-geometry boundary problem for light migration in highly scattering media: A frequency-domain study in the diffusion approximation," JOSA $B$ 11(10), 2128-2138 (1994).

37. T. J. Huppert, S. G. Diamond, M. A. Franceschini, D. A. Boas, "HomER: A review of time-series analysis methods for near-infrared spectroscopy of the brain," Appl. Opt. 48(10), D280-D298 (2009).

38. P. Van der Zee, M. Cope, S. Arridge, M. Essenpreis, L. Potter, A. Edwards, J. Wyatt, D. McCormick, S. Roth, E. Reynolds, Experimentally measured optical pathlengths for the adult head, calf and forearm and the head of the newborn infant as a function of inter optode spacing, Oxygen Transport to Tissue XIII, pp. 143-153, Springer (1992).

39. A. Duncan, J. H. Meek, M. Clemence, C. E. Elwell, L. Tyszczuk, M. Cope, D. T. Delpy, "Optical pathlength measurements on adult head, calf and forearm and the head of the newborn infant using phase resolved optical spectroscopy," Phys. Med. Biol. 40, 295 (1995).

40. F. Felici, V. Quaresima, L. Fattorini, P. Sbriccoli, G. Filligoi, M. Ferrari, "Biceps brachii myoelectric and oxygenation changes during static and sinusoidal isometric exercises," Kinesiology 19(2), 1-11 (2009).

41. B. Molavi, G. Dumont, B. Shadgan, Motion artifact removal from muscle NIR Spectroscopy measurements, 23rd Canadian Conf. Electrical and Computer Engineering (CCECE), pp. 1-4, Calgary (2010).

42. M. Praagman, H. Veeger, E. Chadwick, W. Colier, F. can der Helm, "Muscle oxygen consumption, determined by NIRS, in relation to external force and EMG," J. Biomech. 36, 905-912 (2003).

43. H. Miura, H. Araki, H. Matoba, K. Kitagawa, "Relationship among oxygenation, myoelectric activity, and lactic acid accumulation in vastus lateralis muscle during exercise with constant work rate," Int. J. Sports Med. 21(3), 180-184 (2000).

44. J. Taelman, J. Vanderhaegen, M. Robjins, G. Naulaers, A. Spaepen, S. Van Huffel, "Estimation of muscle fatigue using surface electromyography and near-infrared spectsocopy," Adv. Exp. Med. Biol. 701, 353-359 (2011).

45. J. Cheng, X. Chen, A. Liu, H. Peng, "A novel phonology- and radical-coded Chinese sign language recognition framework using accelerometer and surface electromyography sensors," Sensors (Switzerland) 15, 23303-23324 (2015).

46. H. Dong, I. Ugalde, N. Figueroa, S. Abdulmotaleb , “ Towards whole body fatigue assessment of human movement: A fatigue tracking system based on combined sEMG and accelerometer signals," Sensors 14, 2052-2070 (2014).

47. A. Meigal, S. Rissanen, M. Tarvainen, O. Airaksinen, M. Kankaanpää, P. Karjalainen, "Non-linear EMG parameter for differential and early diagnostics of parkinson's disease," Front. Neurol. 4, article 135 (2013).

48. S. Roy, M. Cheng, S. Chang, J. Moore, G. De Luca, S. Nawab, C. De Luca, "A combined sEMG and accelerometer system for monitoring functional activity in stroke," IEEE Trans. Neural Syst. Rehabil. Eng. 17, 585-594 (2014).

49. M. J. Zwarts, M. Keidel, "Relationship between electrical and vibratory output of muscle during voluntary contraction and fatigue," Muscle Nerve 14(8), 756-761 (1991).

50. C. Luca, L. Gilmore, M. Kuznetsov, S. Roy, "Filtering the surface EMG signal: Movement artefact and baseline noise contamination," J. Biomechan. 43, 1583-1579 (2010).

51. M. Raez, M. Hussain, F. Mohd-Yasin, "Techniques of EMG signal analysis: Detection, processing, 
classification and applications," Biol. Proced. Online 8, 11-35 (2006).

52. J. Virtanen, T. Noponen, K. Kotilahti, J. Virtanen, R. Ilmoniemi, "Accelerometer-based method for correcting signal baseline changes caused by motion artifacts in medical near-infrared spectroscopy," J. Biomed. Opt. 16(8), 087005-1-087005-9 (2011).

53. Patent: http://www.google.ch/patents/US2015016 5269 . 